\title{
Continuous erector spinae plane block is a valid postoperative analgesic alternative in various surgical procedures
}

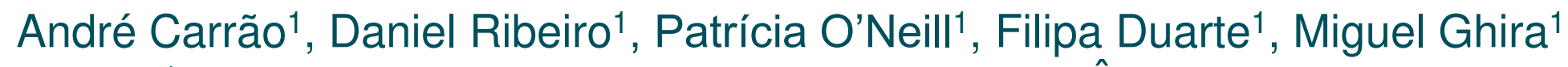

${ }^{1}$ Anesthesiology Department, Hospital Beatriz Ângelo, Portugal

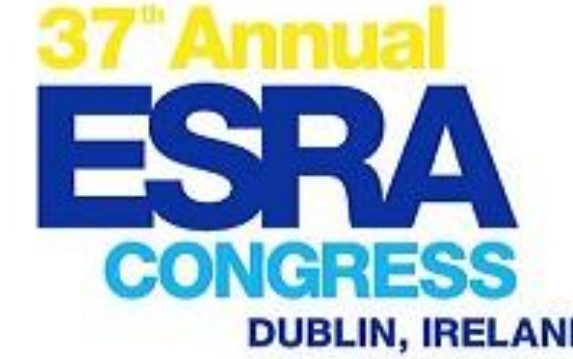

DUBLIN, IRELAND

12-15 SEPTEMBER 2018

\section{Introduction}

The thoracic erector spinae plane block was first described by Forero et al in September 2016 as an option for the control of thoracic neuropathic pain as well as post-operative thoracic pain ${ }^{1}$. Since then, there have been multiple reports about the successful use of this block for other surgical procedures, both as a single-shot and as a continuous technique ${ }^{2,3}$. It appears to be a versatile block and to have some benefits, namely reduced number of complications and side effects. The purpose of this study was to review the patients in whom a continuous erector spinae block was performed from September 2017 to April 2018.

\section{Methods}

We performed a retrospective analysis of the electronic health record (including those from the Acute Pain Unit) of the patients who received a continuous erector spinae block for postoperative analgesia, from September 2017 to April 2018. The statistical analysis was done with SPSS (version 23), according to the type of variable.

\section{Results}

We analyzed 37 patients (17 female and 20 male) and the mean \pm standard deviation of age was $61,1 \pm 17,6$ years. The erector spinae plane block was performed in a variety of surgical procedures, as shown in Table 1. Patients received a loading bolus between 20 and $30 \mathrm{ml}$ of ropivacaine $0.5 \%$ and the post-operative analgesic regimen used was mostly a bolus of $10 \mathrm{ml}$ of ropivacaine $0,2 \%$ every 2 hours. Table 2 describes the pain score and side effects up to $48 \mathrm{~h}$ after surgery. Figure 1 illustrates the ultrasound view of the block.

\begin{tabular}{|c|c|}
\hline \multicolumn{2}{|c|}{$\begin{array}{c}\text { Table } 1 \text { - Post-operative analgesia with continuous } \\
\text { erector spinae plane block }\end{array}$} \\
\hline \multicolumn{2}{|l|}{ Surgical specialty - n (\%) } \\
\hline Digestive Surgery & $20(54,1 \%)$ \\
\hline Supra-mesocolic space & $13(65 \%)$ \\
\hline Infra-mesocolic space & $6(30 \%)$ \\
\hline Radical mastectomy & $1(5 \%)$ \\
\hline Plastic Surgery & $2(5,4 \%)$ \\
\hline Latissimus flap breast reconstruction & $1(2,7 \%)$ \\
\hline Nipple-areola reconstruction & $1(2,7 \%)$ \\
\hline Thoracic Surgery & $8(21,6 \%)$ \\
\hline Rib fractures & $2(5,4 \%)$ \\
\hline Modified Ravitch procedure & $1(2,7 \%)$ \\
\hline Atypical resection of the right lower lobe & $3(8,1 \%)$ \\
\hline Debridement of empyema & $2(5,4 \%)$ \\
\hline Orthopedics & $6(16,2 \%)$ \\
\hline Total hip replacement & $4(10,8 \%)$ \\
\hline Osteosynthesis of femoral fracture & $2(5,4 \%)$ \\
\hline Gynecology & $1(2,7 \%)$ \\
\hline Radical mastectomy & $1(2,7 \%)$ \\
\hline \multicolumn{2}{|l|}{ Level of block ${ }^{1}-\mathrm{n}(\%)$} \\
\hline T5 & $6(27,3 \%)$ \\
\hline T9 & $10(45,5 \%)$ \\
\hline L4 & $6(27,3 \%)$ \\
\hline \multicolumn{2}{|l|}{ Continuous catheter management $-\mathrm{n}(\%)$} \\
\hline \multicolumn{2}{|l|}{ Ropivacaine concentration } \\
\hline Ropivacaine $0,15 \%$ & $5(13,5 \%)$ \\
\hline Ropivacaine $0,2 \%$ & $32(86,5 \%)$ \\
\hline \multicolumn{2}{|l|}{ Ropivacaine volume } \\
\hline $10 \mathrm{ml}$ & $30(81,1 \%)$ \\
\hline $5 \mathrm{ml}$ & $2(5,4 \%)$ \\
\hline $7 \mathrm{ml}$ & $2(5,4 \%)$ \\
\hline $8 \mathrm{ml}$ & $3(8,1 \%)$ \\
\hline \multicolumn{2}{|l|}{ Time between ropivacaine administrations } \\
\hline $1 \mathrm{~h}$ & $5(13,5 \%)$ \\
\hline $2 h$ & $23(62,2 \%)$ \\
\hline $3 h$ & $9(24,3 \%)$ \\
\hline Duration of catheter use (days) - median (IQR) & $2(1)$ \\
\hline
\end{tabular}

\section{References}

1. Forero M et al.; The Erector Spinae Plane Block: A Novel Analgesic Technique in Thoracic Neuropathic Pain; Reg Anesth Pain Med; 2016 ; $41: 621-627$

2. Chin KJ, Malhas L, Perlas A; The Erector Spinae Plane Block Provides Visceral Abdominal Analgesia in Bariatric Surgery: A Report of 3 Cases; Reg Anesth Pain Med; 2017; 42: 372:376

3. Restrepo-Garces CE et al; Bilateral Continuous Erector Spinae Plane Block Contributes to Effective Postoperative Analgesia After Major Open Abdominal Surgery: A Case Report; A \& A Case Report; 2017; 9: 319-321

\begin{tabular}{|c|c|}
\hline \multicolumn{2}{|l|}{ Pain on day 1 post-op (0-10) } \\
\hline At rest ${ }^{1}-$ Mean $\pm S D$ & $1,4 \pm 1,7$ \\
\hline With movement ${ }^{2}-$ Mean \pm SD & $4,0 \pm 2,6$ \\
\hline \multicolumn{2}{|l|}{ Pain on day 2 post-op (0-10) } \\
\hline At rest ${ }^{3}$ - Median (IQR) & $0(2)$ \\
\hline With movement ${ }^{4}-$ Mean \pm SD & $2,3 \pm 2,2$ \\
\hline \multicolumn{2}{|l|}{ Pain on day 3 post-op $(0-10)$} \\
\hline At rest ${ }^{5}$ - Median (IQR) & $0(2)$ \\
\hline With movement ${ }^{6}-$ Mean \pm SD & $2,5 \pm 2,4$ \\
\hline \multicolumn{2}{|l|}{ Post-operative side effects - n (\%) } \\
\hline Nausea and/or vomiting & $5(13,5 \%)$ \\
\hline Paresthesia & $1(2,7 \%)$ \\
\hline No side effects & $2(5,4 \%)$ \\
\hline
\end{tabular}

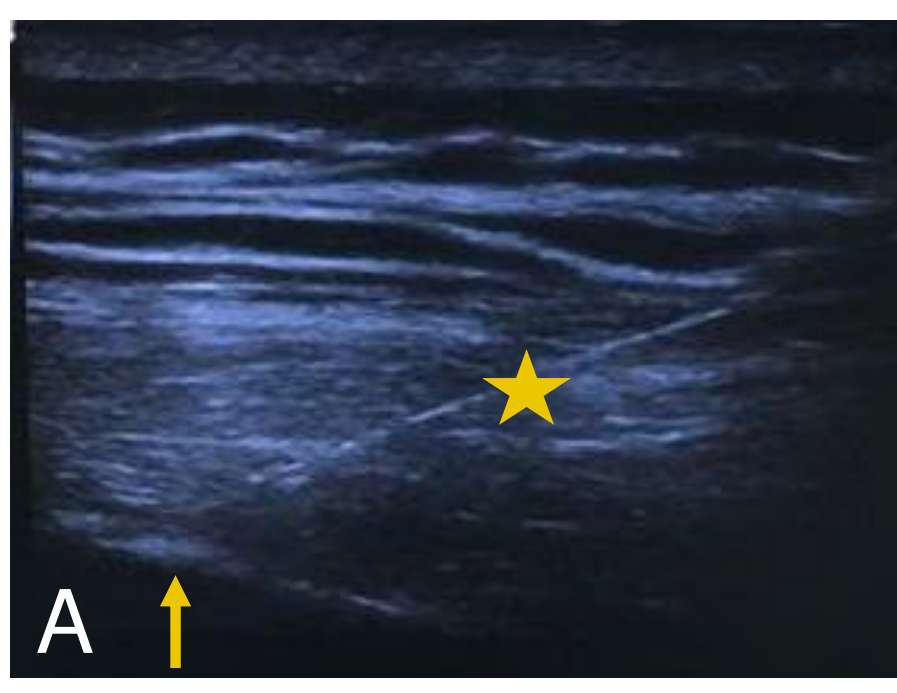

Figure 1 - A) Needle in the erector spinae plane. B) Local anaesthetic spread below erector spinae muscle. Needle (star), transverse process (arrow), local anaesthetic (arrow head)

\section{Conclusion}

This analysis shows that post-operative analgesia with continuous erector spinae plane block can be a valid choice for various surgical procedures. Cumulative evidence of good analgesic efficacy, safety profile and ease of handling could prove this block to be an attractive alternative when neuraxial blockade is not deemed suitable or easily manageable.

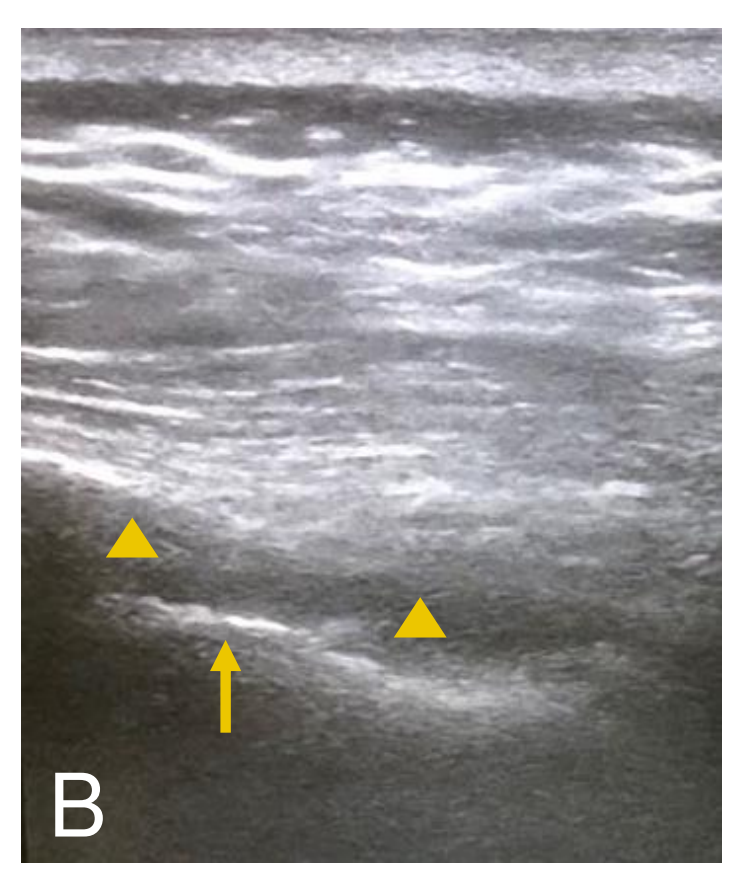

